\title{
Spoof Plasmons Affected by the Corrugated Structures
}

\author{
T. GRIC* \\ Vilnius Gediminas Technical University, Vilnius, Lithuania and \\ Semiconductor Physics Institute, Center for Physical Sciences and Technology, Vilnius, Lithuania
}

(Received 19 December, 2016; in final form 9 August, 2017)

As reported by Ritchie, the fundamental optical excitations that are confined to a metal/dielectric interface are referred as the surface plasmon polaritons. In the present study, we report on a theoretical investigation of the dispersion relation of surface plasmon polaritons on the periodically corrugated surfaces. Tunability of these structures can be enhanced further changing the employed material.

DOI: 10.12693/APhysPolA.132.1347

PACS/topics: 43.35.Pt, 42.70.Nq, 61.82.Fk

\section{Introduction}

As reported by Ritchie [1], the fundamental optical excitations that are confined to a metal/dielectric interface are referred as the surface plasmon polaritons (SPPs). The application of the SPPs in biosensors [2], subwavelength optics, data storage, light generation [3], and nano-imaging [4] open the wide avenues for researchers. In recent times, an idea of engineering surface plasmons at lower frequencies was suggested. Based on the conclusions in [5], the presence of holes in the structure can lower the frequency of existing surface plasmons. Hence, by cutting holes or grooves in metal surfaces, it is possible to deal with concepts such as highly localized waveguiding [6] and superfocusing [7] to lower frequencies, particularly to the $\mathrm{THz}$ regime [8], where plasmonics could enable near-field imaging and biosensing [9] with unprecedented sensitivity. It should be pointed out that in case of the period and width of the grooves being much smaller than the wavelength of excitation, it is possible to replace the perforated structure by a material whose plasma frequency depends on the depth of the grooves and the period of the structure [5] and to apply the effective medium approximation. By this reason, the terminology of "spoof surface plasmons" is applied for the bound surface waves propagating along the perforated structures [10]. Consequently, corrugated surfaces support surface waves named as spoof SPPs, having the behavior depending mainly on the geometry of the corrugations instead of the optical properties of the material [11].

In the present study, we report on a theoretical investigation of the dispersion relation of SPPs on the periodically corrugated surfaces. Dealing with the semiconductor case, we assumed Drude's permittivity model of the semiconductor, which accurately describes the loss of these spoof SPPs. In the $\mathrm{THz}$ frequency range, we have investigated the properties of the dispersion and loss

*corresponding author; e-mail: Tatjana.Gric@dal.ca of spoof SPPs on corrugated Si surfaces. Consequently, a low-loss propagation of spoof SPPs can be achieved providing an optimum design of the surface structure. It was found that a low guiding attenuation can be attained by increase of the lattice constant or by reducing the groove depth. However, nowadays there is a need to search for better materials aimed for plasmonic and metamaterial applications. Transparent conducting oxides (TCOs) are known as low-loss plasmonic materials in the near-infrared wavelength range. The further design of the lower-loss materials would be available by fulfilling a more sophisticated theoretical study. Herein, we address the spoof plasmons on a periodically corrugated surface made of the transparent conducting oxides. We assumed the Drude-Lorentz permittivity model of the semiconductor, which accurately describes the loss of SPPs. In the $\mathrm{THz}$ frequency range the properties of the dispersion and loss of spoof SPPs on corrugated $\mathrm{ZnO}$ and indium tin oxide surfaces are considered. Moreover, to obtain the eigenmodes of the electromagnetic field at a periodically corrugated metamaterial we consider a theory of spoof plasmons propagating on metamaterial perforated with planar periodic grooves. We present the rigorous modeling and analysis of surface waves at the boundary of the corrugated metamaterial structure. Particularly, we pay special attention to the ways of controlling the properties of surface waves. Furthermore, we analyze the dependence of the dispersion characteristics on the metamaterial design.

\section{Propagation of plasmons in corrugated structures}

Consider a corrugated surface in which an array of grooves exists. This structure is shown in Fig. 1. The surface is continuous in the $x$-direction and there are two different materials in the $z-y$ plane: the certain material occupies the lower region and above there is a dielectric as a surrounding medium. Such an interface can be modeled as a three-layer structure, consisting of a homogeneous anisotropic layer of a thickness $h$ - see Fig. 1 describing corrugations, placed between a material and a dielectric. The approximation of the central layer as 


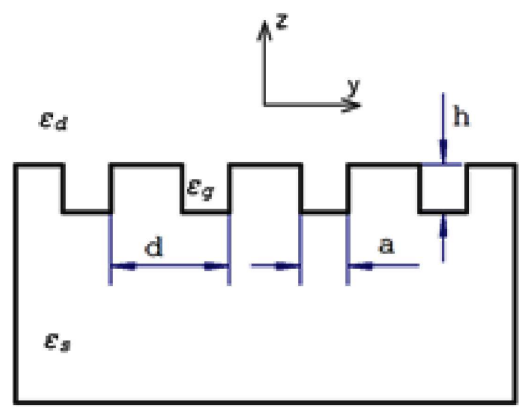

Fig. 1. Geometry of structured surface.

an anisotropic effective medium is possible as long as the period $d$ is much smaller than the wavelength of the electromagnetic field.

In order to find the dielectric parameters of the effective medium, consider a periodic assembly of parallel plates. The effective dielectric constants of such an assembly are as follows [12]:

$$
\begin{aligned}
& \varepsilon_{x}=\varepsilon_{z}=\frac{(d-a) \varepsilon_{s s}+a \varepsilon_{g}}{d}, \\
& \varepsilon_{y}=\frac{d}{(d-a) / \varepsilon_{s s}+a / \varepsilon_{g}} .
\end{aligned}
$$

Here, $\varepsilon_{d}$ is the permittivity of the surrounding media, $\varepsilon_{g}$ is the permittivity of the material, filling the grooves, $\varepsilon_{s s}$ is the permittivity of the structured surface.

By means of the effective medium approximation (Eqs. (1),(2)), the transverse magnetic spoof plasmon mode, propagating in the structure can be described. The only non-zero components of the magnetic and electric fields are $H_{x}, E_{y}, E_{z}$ :

$$
\begin{aligned}
& H_{x}=A \mathrm{e}^{\mathrm{i} k y} \begin{cases}\mathrm{e}^{-\kappa z}, & z>0, \\
\frac{\cos \left(k_{g}(z+h)\right)}{\cos \left(k_{g} h\right)}, & -h<z<0,\end{cases} \\
& E_{y}=-\frac{\partial_{z} H_{x}}{\mathrm{i} k_{0} \varepsilon_{y}}= \\
& -\frac{A \mathrm{i} \mathrm{e}^{\mathrm{i} k y}}{k_{0}} \begin{cases}\frac{\kappa}{\varepsilon_{d}} \mathrm{e}^{-\kappa z}, & z>0, \\
\frac{k_{g}}{\varepsilon_{y}} \frac{\sin \left(k_{g}(z+h)\right)}{\cos \left(k_{g} h\right)}, & -h<z<0,\end{cases} \\
& E_{z}=\frac{\partial_{y} H_{x}}{\mathrm{i} k_{0} \varepsilon_{z}}=\begin{array}{ll}
\frac{A k \mathrm{e}^{\mathrm{i} k y}}{k_{0}} \begin{cases}\frac{1}{\varepsilon_{d}} \mathrm{e}^{-\kappa z}, & -h<z<0 . \\
\frac{\cos \left(k_{g}(z+h)\right)}{\varepsilon_{z} \cos \left(k_{g} h\right)}, & -h<0,\end{cases}
\end{array}
\end{aligned}
$$

Here $k$ is the spoof plasmon wave vector, $k_{g}$ is the wave vector of the wave propagating on the grooves defined as [13]:

$$
k_{g}=k_{0} \sqrt{\varepsilon_{g}}\left(1+\frac{l_{s}(i+1)}{a}\right)^{-1},
$$

where $l_{s}=\left(k_{0} \operatorname{Re} \sqrt{-\varepsilon_{s}}\right)^{-1}$ is the skin depth, $\kappa=$ $\sqrt{k^{2}-\varepsilon_{d} k_{0}^{2}}, A$ is the amplitude, $k_{0}=2 \pi f / c$ is the vacuum wave vector, $h$ is the groove depth in the semicon- ductor.

By expanding the spoof plasmon wave vector presented in [14], i.e. rearranging it in terms of dielectric parameters of the effective medium and matching the fields at the boundary $z=0$, one can obtain the following expression:

$$
k=\sqrt{\varepsilon_{d} k_{0}^{2}+\left(\frac{\varepsilon_{d}}{\varepsilon_{y}}\right)^{2} k_{g}^{2} \tan ^{2}\left(k_{g} h\right)} .
$$

\section{Numerical analysis of dispersion characteristics}

Herein we discuss the numerical solution to the dispersion relation (3), obtained for the structured surface made of semiconductor [15], transparent conducting oxides TCO [16], or metamaterial.

\subsection{Semiconductor case}

The dispersion curves of spoof SPPs on structured surfaces with $d=10 \mu \mathrm{m}$ are shown in Fig. 2a. It is of particular interest to analyze the effect of the groove depth on the dispersion curves of spoof SPPs. For this reason two different groove depths, i.e. $h=3 d$ and $h=2.5 d$ are suggested for the study. It should be pointed out that several values of the groove width are considered for each groove depth. The losses of these spoof SPPs as a function of frequency are presented in Fig. 2b. Compared to a metal $\mathrm{THz}$ waveguide described in [17], the corrugated semiconductor surface exhibits relatively high loss for guiding $\mathrm{THz}$ wave. It is worthwhile mentioning that the frequency ranges of surface wave can be tuned by changing the geometrical dimensions of the structure under consideration. As seen from Fig. 2a, the smallest asymptotic frequency is achieved in case of $a=0.8 d$, $h=3 d$.
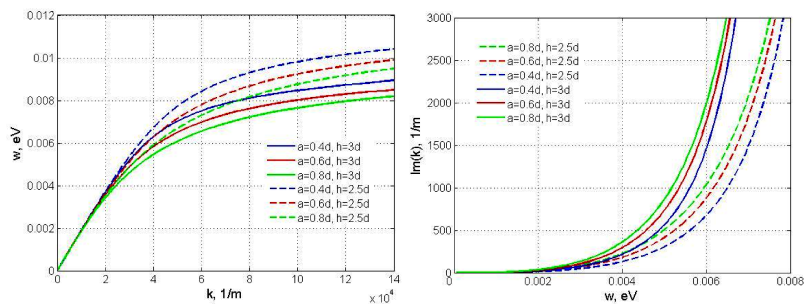

Fig. 2. (a) Dispersion curves for spoof SPPs. (b) Attenuation coefficients of spoof SPPs.

The analysis of the effect of the lattice constant $(d)$ on the dispersion of spoof SPPs is of particular importance. Figure 3a shows the dispersion curves for spoof SPPs on corrugated surfaces with different lattice constants $d=$ 5,7 , and $10 \mu \mathrm{m}$, respectively. The groove parameters are $a=2 \mu \mathrm{m}$ and $h=30 \mu \mathrm{m}$ for all cases. The losses of spoof SPPs for three cases are plotted in Fig. 3b. The former tenability property suggests that the surface wave can be engineered by the either the lattice constant or the period of the structure. 

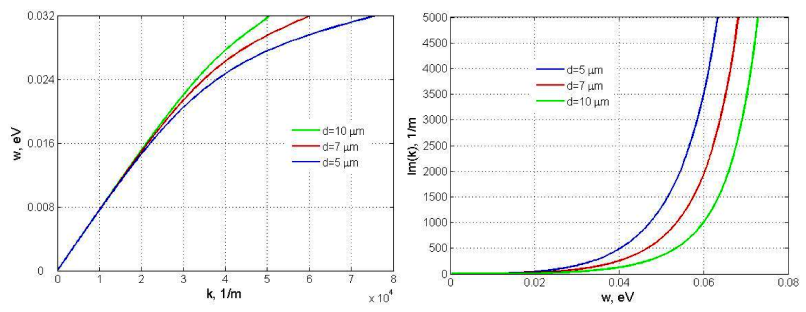

Fig. 3. Dispersion curves (a) and attenuation coefficients (b) of spoof SPPs for different lattice constants $d=5,7$, and $10 \mu \mathrm{m}$, respectively. Parameters of grooves $a=2 \mu \mathrm{m}$ and $h=30 \mu \mathrm{m}$.

\subsection{TCO case}

In this section, a simple example of polaritons in corrugated oxide semiconductors at $\mathrm{THz}$ frequencies is given. The case of $\mathrm{ZnO}$ (AZO) is considered. Herein, we analyzed the evolution of the dispersion relation of the surface EM waves supported by this structure with groove depth and width. As seen from Fig. 4a, the asymptotic
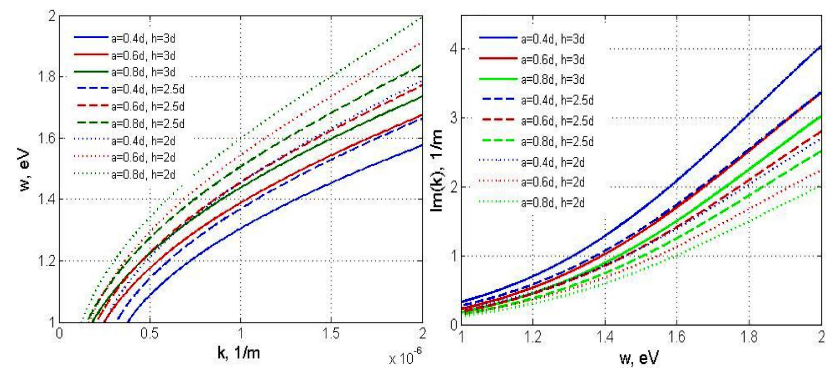

Fig. 4. (a) Dispersion curves for spoof SPPs. (b) Attenuation coefficients of spoof SPPs. Lattice constant $d=1 \mathrm{~nm}$.

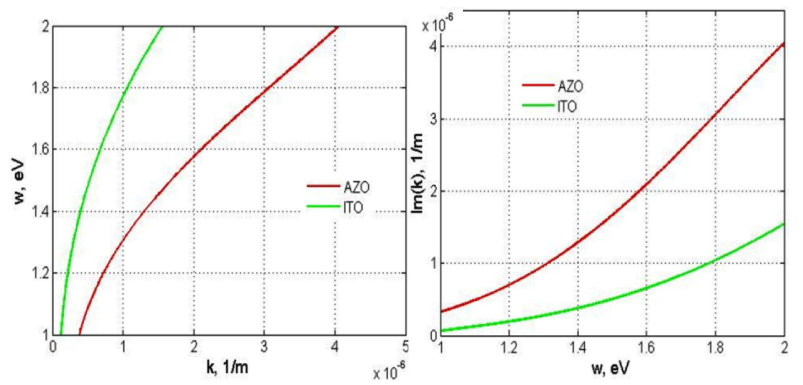

Fig. 5. Dispersion curves (a) and attenuation coefficients (b) of spoof SPPs for different materials, i.e. AZO and ITO, respectively. Parameters of grooves $a=0.4 \mathrm{~nm}$ and $h=1.2 \mathrm{~nm}$.

frequency decreases by increase of the groove depth and decrease of the groove width. From Fig. $4 \mathrm{~b}$ it can be observed that the loss of spoof SPPs grows significantly with increase of the frequency. Compared to a semiconductor structure described in [15], the corrugated transparent conducting oxide surface exhibits relatively low loss for guiding the wave. The mentioned outcome appears due to the fact that the permittivity of the transparent conducting oxide is smaller than that of silicon.

To study the material effect on the spoof SPP wave propagation, we calculated the dispersion characteristics for different materials. Figure 5a shows the dispersion curves for spoof SPPs on corrugated surfaces with different materials, i.e. AZO and indium tin oxide (ITO), respectively. The groove parameters are $a=0.4 \mathrm{~nm}$ and $h=1.2 \mu \mathrm{m}$ for all cases. The losses of spoof SPPs for two cases are plotted in Fig. 5b. As seen from Fig. 5b, a material with a higher permittivity, i.e. AZO corresponds to a larger loss of spoof SPPs for a given frequency.

\subsection{Metamaterial case}

In order to understand the properties of the reported SPPs, we provide a simple example of polaritons in corrugated metamaterials at $\mathrm{THz}$ frequencies. The dispersion curves of spoof SPPs on structured surfaces with $d=200 \mu \mathrm{m}$ are shown in Fig. 6a.
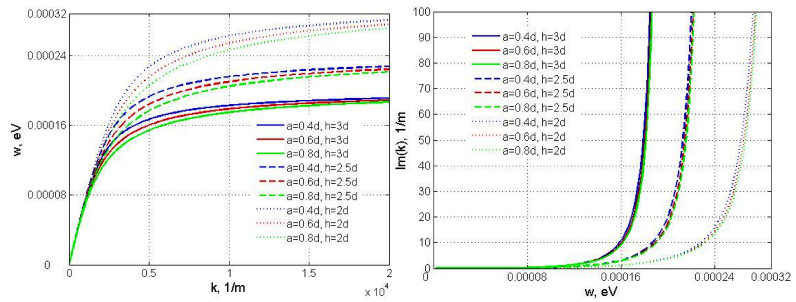

Fig. 6. (a) Dispersion curves for spoof SPPs. (b) Attenuation coefficients of spoof SPPs, lattice constant $d=200 \mu \mathrm{m}$.

It is worthwhile mentioning that the plasma frequency of the considered surface waves is drastically affected by the chosen material. Thus the smallest value is achieved in the metamaterial case while the largest one in case of the TCO.

\section{Conclusions}

A method for analyzing spoof SPPs on a periodically corrugated surfaces has been presented. This work aims at providing a comprehensive and updated picture of a dynamic control of the dispersion diagrams, extremely desirable in view of device production. We provided the dispersion relation, and discussed the controllable properties of the dispersion diagrams. Compared to the previous works, our approach enables us to investigate different cases since the models of all the materials under consideration might be included into the analysis. Moreover, the simulation results of the corrugated metamaterials corroborate the conclusions obtained through an analysis based on the study of isotropic semiconductor grating interface [15] and allow us to provide the vivid demonstrations of phenomena occurring in metamaterial 
interfaces, such as the decrease of the asymptotic frequency. The proposed theory may give the rise to the investigation of other important propagation characteristics of spoof plasmons such as quality factor and propagation length. It is also of the potential interest to consider $\mathrm{THz}$ field confinement by adiabatic increase of the depth of the grooves.

\section{References}

[1] R.H. Ritchie, Phys. Rev. 106, 874 (1957).

[2] S.A. Maier, H.A. Atwater, J. Appl. Phys. 98, 011101 (2005).

[3] W.L. Barnes, A. Dereux, T.W. Ebbesen, Nature 424, 824 (2003).

[4] N. Fang, H. Lee, C. Sun, X. Zhang, Science 308, 534 (2005).

[5] J.B. Pendry, L. Martın-Moreno, F.J. Garcia-Vidal, Science 305, 847 (2004).

[6] S.A. Maier, P.G. Kik, H.A. Atwater, S. Meltzer, E. Harel, B.E. Koel, A.A.G. Requicha, Nat. Mater. 2, 229 (2003).

[7] M.I. Stockman, Phys. Rev. Lett. 93, 137404 (2011).
[8] B. Ferguson, X.-C. Zhang, Nat. Mater. 1, 26 (2002).

[9] M. Nagel, P. Haring Bolivar, M. Brucherseifer, H. Kurz, A. Bosserhoff, R. Büttner, Appl. Phys. Lett. 80, 154 (2002).

[10] A.I. Fernandez-Dominguez, L. Martin-Moreno, F.J. Garcia-Vidal, S.R. Andrews, S.A. Maier, IEEE J. Sel. Top. Quantum Electron. 14, 1515 (2008).

[11] D. Woolf, M.A. Kats, F. Capasso, Opt. Lett. 39, 517 (2014).

[12] M. Born, E. Wolf, Principles of Optics, Cambridge University Press, Cambridge 1999.

[13] A. Rusina, M. Durach, K.A. Nelson, M.I. Stockman, Opt. Expr. 16, 18576 (2008).

[14] A. Rusina, M. Durach, M.I. Stockman, Appl. Phys. A 100, 375 (2010).

[15] T. Gric, M.S. Wartak, M. Cada, J.J. Wood, O. Hess, J. Pistora, J. Electromagnet Wave 29, 1899 (2015).

[16] T. Gric, J. Electromagnet Wave 30, 721 (2016).

[17] L. Shen, X. Chen, T.-J. Yang, Opt. Express 16, 3326 (2008). 